\title{
Improving Bahr's invariant parameters using the WAL approach
}

\author{
Anna Martí, ${ }^{1}$ Pilar Queralt, ${ }^{1}$ Alan G. Jones ${ }^{2}$ and Juanjo Ledo ${ }^{1}$ \\ ${ }^{1}$ Departament de Geodinàmica i Geofisica, Universitat de Barcelona, C/ Martí i Franquès s/n, 08028 Barcelona, Spain. E-mail: annamarti@ub.edu \\ ${ }^{2}$ Dublin Institute for Advanced Studies, 5, Merrion Square, Dublin 2, Ireland
}

Accepted 2005 July 12. Received 2005 May 30; in original form 2004 November 3

\begin{abstract}
SUMMAR Y
In the magnetotelluric technique, several methods exist to perform dimensionality analysis of the measured data using rotational invariants of the impedance tensor. Among these methods there is some dilemma on the different criteria established, which sometimes lead to nonequivalent interpretations. The aim of this work is to compare the Bahr and Weaver et al. (WAL hereafter) methods, and to propose a new method that makes both dimensionality methods consistent. This new method complements the parameters used in Bahr method with WAL invariant $Q$, and redefines their threshold values. To accomplish this, we used the analytical relations between both sets of parameters and re-analyse and compare the threshold values of each method. Both the Bahr and WAL methods use sets of rotational invariant parameters of the magnetotelluric tensor $\left[\underline{\boldsymbol{M}}\left(\mathrm{m} \mathrm{s}^{-1}\right) ; \vec{E}=\underline{\boldsymbol{M}} \cdot \vec{B}\right]$ and establish a classification of their values to determine the kind of dimensionality associated to the measured data.
\end{abstract}

Key words: geoelectric dimensionality, magnetotelluric tensor, rotational invariants, threshold values.

\section{BAHR METHOD}

Bahr (1991) (with modifications of Szarka 1999) was the first author who presented a classification of the types of distortion that affect the regional structures by quantifying the values of four rotational invariant parameters: $\kappa$ (swift skew), $\mu, \eta$, (regional skew of phase sensitive skew) and $\Sigma$. Bahr parameters are dimensionless; $\mu$ and $\eta$ are normalized to unity whereas $\kappa$ and $\Sigma$ can have values greater than 1 in the presence of galvanic distortion. $\kappa$ is related to one-dimensionality. $\mu$ is a measure of the phase difference in the magnetotelluric tensor. $\eta$ indicates if the magnetotelluric tensor can be described by a superimposition model (a real distortion matrix that represents a 3-D small heterogeneity producing galvanic distortion multiplying the regional 1-D or 2-D magnetotelluric tensor: $3-\mathrm{D} / 1-\mathrm{D}$ or 3-D/2-D) and is also a measure of three-dimensionality. $\Sigma$ is related to two-dimensionality. The information given by these parameters, and the recommended threshold values according to Bahr (1991), are summarized in Table 1.

One of the main limitations of the Bahr method is that, except for $\kappa$ and $\eta$ (Simpson \& Bahr 2005), the threshold values do not have a justified physical or mathematical meaning, or are set in a statistical framework. As we will show below, the use of only these four parameters is insufficient to characterize completely the dimensionality (Ledo et al. 2002).

\section{WAL METHOD}

Weaver et al. (2000), following and extending the work of Szarka \& Menvielle (1997), defined a set of seven independent $\left(I_{1}-I_{7}\right)$ rotational invariant parameters. An eight invariant, dependent on the other ones, $Q$, is also defined, which controls the value of $I_{7}$. As $Q$ approaches zero, that is, for error-free and distortion free data from a 1-D or 2-D Earth, then $I_{7}$ approaches infinity and its value is undetermined.

Invariants $I_{1}$ and $I_{2}$ provide information about the 1-D magnitude and phase of the geoelectrical resistivity. The other invariants characterize the dimensionality according to whether their values are null of not (Table 1). Cases 3 and 4 are related to different types of galvanic distortion. Case 3a (3-D/2-Dtwist) corresponds to a local distortion caused by a twist of the electric field (Groom \& Bailey 1989).

Case $3 \mathrm{~b}$ corresponds to a regional 1-D or 2-D with equal phases for both $x y$ and $y x$ polarizations, affected by small-scale distortion (3-D/2-D1-D). In this case there is an ambiguity in the recovery of the regional tensor (Weaver et al. 2000). Case 4 corresponds to a regional 2-D tensor affected by galvanic distortion described by a general distortion tensor (3-D/2-D) (Groom \& Bailey 1989).

For real data, it is necessary to use a threshold value to evaluate whether an invariant is null or not.

\section{COMPARISON BETWEEN BOTH METHODS}

The number of WAL invariants used for determining dimensionality criteria is six $\left(I_{3}-I_{7}\right.$ and $\left.Q\right)$, which can be reduced to five since $I_{3}$ and $I_{4}$ are used together in the dimensionality classification. 
Table 1. Dimensionality cases according to Bahr and WAL rotational invariants methods.

\begin{tabular}{|c|c|c|c|}
\hline Bahr & WAL & & Dimensionality cases \\
\hline$\kappa<0.1, \Sigma<0.1$ & $\begin{array}{l}I_{k}=0(k=3-6), \\
I_{7}=0 \text { or } Q=0\end{array}$ & & 1) $1-\mathrm{D}$ \\
\hline$\kappa<0.1, \Sigma>0.1$ & $\begin{array}{c}I_{3} \neq 0 \text { or } I_{4} \neq 0, \\
I_{7}=0 \text { or } Q=0\end{array}$ & & 2) $2-\mathrm{D}$ \\
\hline$\kappa>0.1, \mu=0$ & $\begin{array}{l}I_{3} \neq 0 \text { or } I_{4} \neq 0 \\
\quad \text { and } I_{5} \neq 0\end{array}$ & $\begin{array}{l}\text { WAL } \\
\text { Bahr }\end{array}$ & $\begin{array}{c}\text { 3a) 3-D } / 2 \text {-Dtwist }\left(I_{7}=0\right) \\
\text { 3b) } 3 \text {-D } / 2-\mathrm{D} 1-\mathrm{D} \\
\left(I_{7} \text { undefined }\right) \\
\text { 3) } 3 \text {-D } / 1-\mathrm{D}\end{array}$ \\
\hline$\kappa>0.1, \mu \neq 0, \eta<0.05$ & $I_{6} \neq 0$ & & 4) $3-\mathrm{D} / 2-\mathrm{D}$ \\
\hline$\kappa>0.1, \mu \neq 0, \eta>0.3$ & $I_{7} \neq 0$ & & 5) 3-D \\
\hline
\end{tabular}

In order to compare the reliability of both methods, we used an example of a magnetotelluric tensor $\underline{\boldsymbol{M}}$, clearly representing a 2-D structure, with different phases in the $x y$ and $y x$ polarizations: $M=\left[\begin{array}{cc}0 & 25+9 i \\ -15-12 i & 0\end{array}\right]$. Bahr parameters values are $\kappa=0$, $\mu=0, \eta=0$ and $\Sigma=0.05$. According to Bahr criteria (Table 1: $\kappa$ and $\Sigma<0.1$, and $\eta$ and $\mu \approx 0$ ), this values would correspond to a 1-D or 3-D/1-D structure. WAL invariant values $I_{3}-I_{7}$ and $Q$ are $I_{3}=0.25, I_{4}=0.14, I_{5}=I_{6}=I_{7}=0$ and $Q=0.39$. Following WAL criteria, this MT tensor represents a 2-D structure.

From these different dimensionality interpretations, it is clear that, in some circumstances, Bahr's criteria lead to incorrect inferences.

The use of only four parameters in the Bahr method is an important limitation in determining dimensionality, but also the classification of the dimensionality types and the threshold values should be revised to become more concise and consistent.

\section{ANALYTICAL RELATIONSHIPS AND EQUIVALENCIES FOR IDEAL CASES}

The analytical relationships were obtained by expressing Bahr parameters as functions of WAL invariants and are given as:

$$
\begin{aligned}
& \kappa=f\left(r, I_{5}, I_{6}\right)=\sqrt{\frac{1+r^{2}}{1-\frac{a}{2 b}+\frac{r^{2} b}{2}}-1}, \\
& \mu=f\left(r, I_{5}, I_{6}, I_{7}, Q\right)=\sqrt{\frac{\left|I_{7} Q+I_{6}\right|+\left|I_{6}\right|}{\frac{1}{r}\left(1-\frac{a}{2 b}\right)+\frac{r b}{2}}}, \\
& \eta=f\left(r, I_{5}, I_{6}, I_{7}, Q\right)=\sqrt{\frac{\left|I_{7} Q\right|}{\frac{1}{r}\left(1-\frac{a}{2 b}\right)+\frac{r b}{2}}}, \\
& \Sigma=f\left(r, I_{3}, I_{4}, I_{5}, I_{6}\right)=\frac{I_{3}^{2}+I_{4}^{2} r^{2}}{1-\frac{a}{2 b}+\frac{r^{2} b}{2}},
\end{aligned}
$$

where $a$ and $b$ depend on $I_{5}$ and $I_{6}$ :

$a=\left(I_{5}-I_{6}\right)^{2}, b=1-I_{5} I_{6}+\sqrt{1+I_{5}^{2} I_{6}^{2}-I_{5}^{2}-I_{6}^{2}}$.

$r$ is the quotient between $I_{2}$ and $I_{1}$, which appears in all the relations due to the different kinds of normalization used in each set of parameters.

Relating these identities to WAL dimensionality criteria, parameter $k$ is the one that distinguishes between undistorted and distorted cases (depending on $I_{5}$ and $I_{6}$ being null or not). $\mu$ and $\eta$ depend also on the product $I_{7} \cdot Q$, but not on their individual values; and
$\Sigma$ is the only one that depends on $I_{3}$ and $I_{4}$, and, consequently, that deals with two-dimensionality.

The analytical relations presented allow finding easily the particular expressions of Bahr parameters for the ideal cases following the WAL conditions (Table 1):

(1) 1-D: $\kappa=\mu=\eta=\Sigma=0$.

(2) 2 -D: $\kappa=\mu=\eta=0$ and $\Sigma=f\left(r, I_{3}, I_{4}\right)=\frac{I_{3}^{2}+I_{4}^{2} r^{2}}{1+r^{2}}$.

(3) 3a) and 3b) (3-D/2-Dtwist) and (3-D/2-D1-D): $\mu=\eta=$ $0, \kappa=f\left(r, I_{5}\right)$ and $\Sigma=f\left(r, I_{3}, I_{4}, I_{5}\right)$. $3 \mathrm{a}$ and $3 \mathrm{~b}$ are nondistinguishable using the Bahr method because it is not possible to know which of $I_{7}$ and $Q$ is null.

(4) 3-D/2-D: $\eta=0$ and $\kappa, \mu$ and $\Sigma$ take the values corresponding to the general expressions)

(5) 3-D: $\kappa, \mu, \eta$ and $\Sigma$ take the values corresponding to the general expressions (eqs 1a-1d).

From these relations some important points arise that change the Bahr criteria given in Table 1:

(a) If the regional structure is $1-\mathrm{D}$ or $2-\mathrm{D}$, parameter $\mu$ must be null.

(b) $\kappa \neq 0$ and $\mu \neq 0$ conditions are not necessary for threedimensionality.

(c) Parameter $\eta$ vanishes, as long as the structure is not 3-D.

\section{THRESHOLD VALUES}

Given that in real situations data are affected by noise and that geoelectric structures do not fit exactly the assumed ideal cases, invariant values are never precisely zero. Weaver et al. (2000) address this problem by introducing a threshold value $\left(\tau_{\text {WAL }}\right)$ beneath which invariants $I_{3}-I_{7}$ are considered to be zero.

In this section we present the relations between WAL and Bahr thresholds values, and redefine the ones used in Bahr method in a way that the dimensionality criteria considering the threshold is the same for Bahr and WAL methods.

We performed an statistical approach: For each dimensionality case, random values of the invariants, above or below their corresponding threshold range, $\tau_{\text {WAL }}$, were generated, and Bahr parameters computed using the analytical relations in eqs (1a)-(1d). The value of $r$ was chosen as $r=1$, which is a valid approximation, since for experimental data $I_{1}$ and $I_{2}$ are of the same order of magnitude ( $r$ represents the relation between the imaginary and real parts in the magnetotelluric tensor components). Thus, it was possible to define the new thresholds for Bahr parameters, $\tau_{K}, \tau_{\mu}, \tau_{\eta}$ and $\tau_{\Sigma}$, and the new dimensionality conditions. This development was made for different values of $\tau_{\mathrm{WAL}}$. 
The results show the following:

(1) Thresholds $\tau_{K}$ and $\tau_{\Sigma}$ can easily be related to $\tau_{\text {WAL }}$ using the approaches $\tau_{\kappa} \approx \frac{\tau_{\mathrm{WAL}}}{\sqrt{2}}$ and $\tau_{\Sigma} \approx \tau_{\mathrm{WAL}}^{2}$. The threshold $\tau_{K}$ differentiates 1-D and 2-D from the rest of the cases, excepting 3-D, which can take any value of $\kappa$. Values of $\Sigma$ greater than $\tau_{\Sigma}$ indicate that the structure is not 1-D, but 2-D, with or without galvanic distortion (cases 2-4). 3-D cases can have any value of $\Sigma$.

(2) The dependence of $\tau_{\mu}$ on $\tau_{\mathrm{WAL}}$ is more complex, since these parameters become more sensitive on the value of $r$, and the approximation $r=1$ is not always appropriate.

In order to distinguish between cases $1-3$ from case 4 , there is a statistical value of threshold $\tau_{\mu}$, although sometimes there is an overlap of values among these different cases.

(3) In the case of parameter $\eta$, in order to differentiate between cases 4 and 5 , it is necessary to consider also WAL parameter $Q$, which controls if $I_{7}$ is meaningless or not. This is accomplished assuming that the condition $\eta>\tau_{\eta}$ is valid only if $Q$ is not meaningless, for which case we define the threshold $\tau_{Q}$. The value of $Q$ is also useful to differentiate case $3 a$ from $3 b$, as in the WAL criteria.

Weaver et al. (2000) suggest a threshold value $\tau_{\mathrm{WAL}}=0.1$. Since WAL invariants $I_{3}-I_{7}$ and $Q$ represent the sines of angles related to Mohr circles, this threshold is the sine of $5.7^{\circ}$, which, in relation to a circle, $90^{\circ}$, represents a 6 per cent error.

The threshold values of Bahr's parameters that correspond to $r=$ $1, \tau_{\mathrm{WAL}}=0.1$ and $\tau_{Q}=0.1$ are: $\tau_{K}=0.06, \tau_{\mu}=0.34, \tau_{\eta}=0.12$ and $\tau_{\Sigma}=0.01$. These thresholds, especially $\tau_{\Sigma}$, differ significantly from the thresholds proposed in Bahr (1991).

\section{BAHR- $Q$ METHOD}

From the threshold values for Bahr parameters obtained, we can redefine the appropriate dimensionality conditions. In order to overcome the limitation produced by the use of only four parameters compared to the five of the WAL method, we extend the Bahr method to be internally consistent with WAL invariants.

To classify dimensionality it is necessary to take into account parameters $\kappa, \mu, \eta, \Sigma$ and WAL invariant $Q$, which distinguishes
Table 2. Bahr- $Q$ method criteria to characterize the geoelectric dimensionality.

\begin{tabular}{|c|c|c|c|c|c|}
\hline \multicolumn{6}{|c|}{ B- $Q$ method } \\
\hline Parameter & $\kappa$ & $\mu$ & $\Sigma$ & $\eta$ & $Q$ \\
\hline Threshold $\tau$ & 0.06 & 0.34 & 0.01 & 0.12 & 0.1 \\
\hline 1) $1-\mathrm{D}$ & $<\tau_{\kappa}$ & $<\tau_{\mu}$ & $<\tau_{\Sigma}$ & $\begin{array}{l}<\tau_{\eta} \\
>\tau_{\eta}\end{array}$ & $Q \overline{<} \tau_{Q}$ \\
\hline 2) $2-\mathrm{D}$ & $<\tau_{\kappa}$ & $<\tau_{\mu}$ & $>\tau_{\Sigma}$ & $\begin{array}{l}<\tau_{\eta} \\
>\tau_{\eta}\end{array}$ & $Q \overline{<} \tau_{Q}$ \\
\hline $\begin{array}{l}\text { 3a) 3-D/2-Dtw } \\
\text { 3b) 3-D/1-D2-D }\end{array}$ & $>\tau_{\kappa}$ & $<\tau_{\mu}$ & $>\tau_{\Sigma}$ & $\begin{array}{c}<\tau_{\eta} \\
\text { any value }\end{array}$ & $\begin{array}{l}\text { a) } Q>\tau_{Q} \\
\text { b) } Q<\tau_{Q}\end{array}$ \\
\hline 4) $3-\mathrm{D} / 2-\mathrm{D}$ & $>\tau_{\kappa}$ & $>\tau_{\mu}$ & $>\tau_{\Sigma}$ & $\begin{array}{l}<\tau_{\eta} \\
>\tau_{\eta}\end{array}$ & $Q \overline{<} \tau_{Q}$ \\
\hline 5) $3-\mathrm{D}$ & - & - & - & $>\tau_{\eta}$ & $Q>\tau_{Q}$ \\
\hline
\end{tabular}

the two subclasses of case 3 , and the thresholds as defined in the previous section. We call this new method Bahr- $Q$ (B- $Q$ ), whose conditions are summarized in Table 2 .

The application of B- $Q$ method on the previous example leads to the same interpretation as WAL criteria: $\kappa=\mu=\eta=\Sigma=0 ; \Sigma>$ $\tau_{\Sigma}$ and $Q>\tau_{Q}$, which indicates two-dimensionality.

\section{TEST WITH REAL DATA}

To compare the dimensionality obtained using WAL, Bahr and B- $Q$ methods we used site 85_314 from COPROD2 MT dataset (Jones 1993), commonly used to test and compare 2-D inversion codes. The data responses have in general a 1-D behaviour at short periods (up to $10 \mathrm{~s}$ ). From 10 to $1000 \mathrm{~s}$ the data displays a 2-D behaviour with a NS strike direction (Jones et al. 2005), determined from the multisite-multifrequency decomposition code of McNeice \& Jones (2001), based on Groom \& Bailey (1989).

Table 3 shows Bahr and $Q$ parameters related to the threshold values and the dimensionality derived from the B- $Q$ method and compare to the ones obtained using WAL and Bahr classical criteria. The results show how all methods coincide at short periods (1-D up

Table 3. Dimensionalities obtained from Bahr, WAL and B- $Q$ methods for COPROD2 set, site 314. B- $Q$ methods uses $\tau_{K}, \tau_{\mu}, \tau_{\eta}$ and $\tau_{\Sigma}$ obtained from $\tau_{\mathrm{WAL}}=0.1$, and $\tau_{Q}=0.1$ (Table 2).

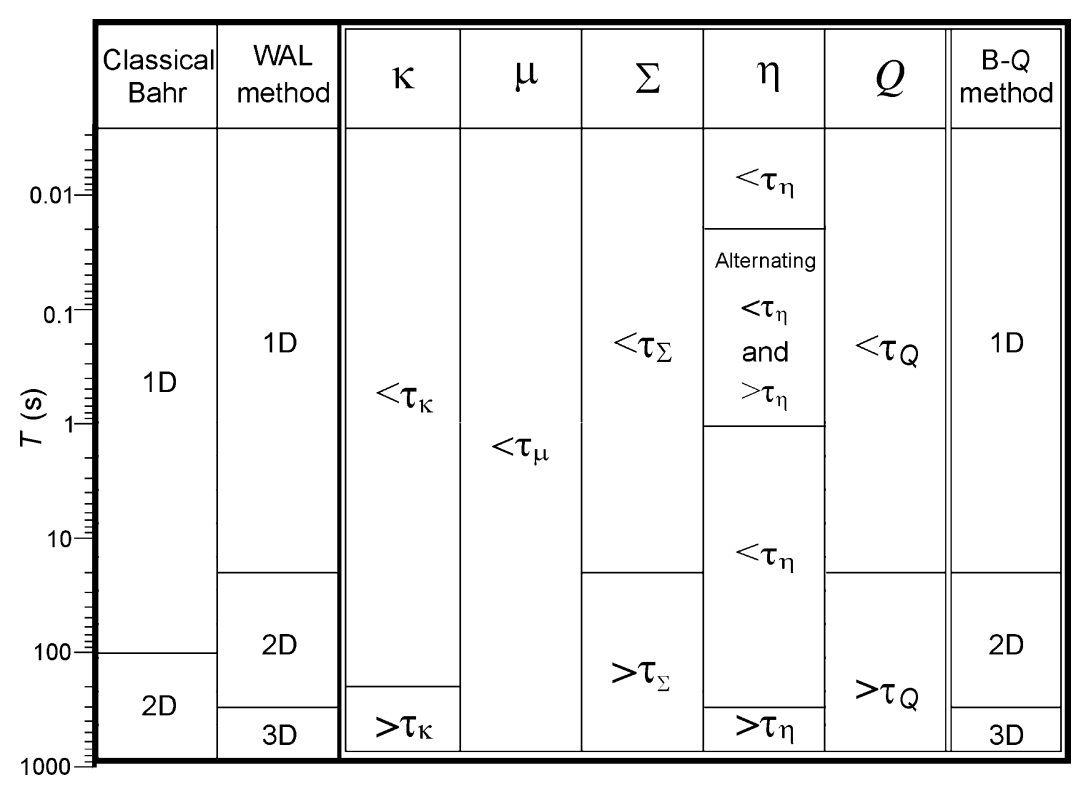


to $10 \mathrm{~s}$ ), whereas for $T>10 \mathrm{~s}$, classical Bahr results differ from B- $Q$ and WAL methods. According to them, the longest periods have a 3-D behaviour, as can be derived from the non-zero values of the diagonal elements of $\underline{\boldsymbol{M}}$ tensor, and consequently from $I_{7}$.

When the data are affected by errors, which affect the computed parameters, one of the consequences is that the dimensionality can be undetermined if the error bars cross the threshold values, because in these cases there are ambiguities in the classification. Another one is the bias that can appear between the true values (computed directly from the tensor components) and the statistical values (computed as the mean of the different Gaussian generations) (Martí et al. 2004).

In this example, for a noise level of 1 per cent, a complete analysis of the dimensionality using WAL and B- $Q$ method would led to a good determination of dimensionality of all the periods. When the noise level is 10 per cent, there are some periods (up to $1.33 \mathrm{~s}$ ) for which $I_{7}$ has large error bars and it is not possible to distinguish if its value is above or below the threshold value. For this reason, the dimensionality results are different for these periods.

\section{CONCLUSIONS}

Compared to WAL, the Bahr method does not provide complete dimensionality interpretations. The reasons are that it uses only four parameters (whereas WAL uses five), and because the thresholds values suggested lead to ambiguities or to situations where the dimensionality cannot be determined.

The redefinition of Bahr values thresholds and the addition of the invariant $Q$ allowed the establishment of a new dimensionality criteria (B- $Q$ ) consistent with WAL method.

The B- $Q$ method was tested using a real example, obtaining the same interpretation as WAL method. When the data are affected by an important level of noise (10 per cent or higher), the errors and biases of the invariants can be important and, consequently, the dimensionality using any of both methods can be undetermined.

\section{ACKNOWLEDGMENTS}

This work was supported by the Ministerio de Ciencia y Tecnología, Spain, project REN2002-04538-C02-01/HID and Acció Integrada Generalitat de Catalunya ACI-2003-8: collaboration between DIAS and UB. The authors acknowledge the reviewers L. Szarka, K. Bahr, H. Bibby and the editor M. Unsworth, for their suggestions and help.

\section{R EFERENCES}

Bahr, K., 1991. Geological noise in magnetotelluric data: a classification of distortion types, Phys. Earth planet. Inter., 66, 24-38.

Groom, R.W. \& Bailey, R.C., 1989. Decomposition of the magnetotelluric impedance tensor in the presence of local three-dimensional galvanic distortion, J. geophys. Res., 94, 1913-1925.

Jones, A.G., 1993. The COPROD2 dataset: tectonic setting, recorded MT data, and comparison of models, J. Geomag. Geoelectr., 45, 933-955.

Jones, A.G., Ledo, J. \& Ferguson, I.J., 2005. Electromagnetic images of the Trans-Hudson orogen: the North American Central Plains (NACP) anomaly revealed, Canadian Journal of Earth Sciences, 42, 457-478.

Ledo, J., Queralt, P., Martí, A. \& Jones, A.G., 2002. Two-dimensional interpretation of three-dimensional magnetotelluric data: an example of limitations and resolution, Geophys. J. Int., 150, 127-139.

Martí, A., Queralt, P. \& Roca, E., 2004. Geoelectric dimensionality in complex geologic areas: application to the Spanish Betic Chain, Geophys. J. Int., 157, 961-974.

McNeice, G. \& Jones, A.G., 2001. Multisite, multifrequency tensor decomposition of magnetotelluric data, Geophysics, 66, 158-173.

Simpson, F. \& Bahr, K., 2005. Practical Magnetotellurics, Cambridge University Press, Cambridge.

Szarka, L., 1999. A correction to Bahr's "phase deviation" method for tensor decomposition. Earth, Planets and Space, 51, 1019-1022.

Szarka, L. \& Menvielle, M., 1997. Analysis of rotational invariants of the magnetotelluric impedance tensor, Geophys. J. Int., 129, 133-142.

Weaver, J.T., Agarwal, A.K. \& Lilley, F.E.M., 2000. Characterisation of the magnetotelluric tensor in terms of its invariants, Geophys. J. Int., 141, $321-336$. 